\title{
Surgeon Oriented Preoperative Radiologic Evaluation in Cochlear Implantation - Our experience with a Proposed Checklist
}

\author{
Mahmoud Mandour $^{1}$ Mohammed Tomoum ${ }^{1}$ Saad El Zayat ${ }^{2}$ Hisham Hamad ${ }^{1}$ Mohamed Amer ${ }^{1}$ \\ ${ }^{1}$ Department of ORL-HNS, Tanta University Faculty of Medicine, \\ Tanta, Egypt \\ 2 Department of ORL-HNS, Kafrelsheikh University, Kafr el-Sheikh, \\ Address for correspondence Mahmoud Mandour, MD, Department of \\ ORL-HNS, Faculty of Medicine, Tanta University, Elgesh Street, \\ Tanta 31111, Egypt (e-mail: mahmoud.mandour@med.tanta.edu.eg).
} Egypt

Int Arch Otorhinolaryngol 2019;23:137-141.

\begin{abstract}
Introduction Preoperative temporal bone imaging studies have been routinely performed prior to cochlear implantation. Radiologists need to report these examinations with special focus on the surgeon's expectations.

Objectives To provide a basic structured format, in the form of a checklist, for reporting preoperative computed tomography (CT) and to its clinical impact on operative findings.

Methods The preoperative temporal bone CT scans of 47 patients were analyzed and reported according to the proposed checklist. Intraoperative assessment of mastoidectomy, posterior tympanotomy and round window access was done by the surgeon in a blinded fashion and were correlated with the radiological findings to assess its significance.

Results The proposed radiological checklist was reliable in assessing operative difficulty during cochlear implantation. Contracted mastoid and lower tegmen posi-

Keywords

- cochlear implant

- cochlear round window

- temporal bone

- round window

- ear tion were associated with a greater difficulty of the cortical mastoidectomy. Presence of an air cell around the facial nerve (FN) was predictive of easier facial recess access exposure. Facial nerve location and posterior external auditory canal (EAC) wall inclination were predictive of difficult round window (RW) accessibility.

Conclusion Certain parameters on the preoperative temporal bone CT scan may be useful in predicting potential difficulties encountered during the key steps involved in cochlear implant surgery.
\end{abstract}

\section{Introduction}

There are three key surgical steps for the cochlear implant; they include 1) cortical mastoidectomy; 2) posterior tympanotomy; and 3) round window identification and cochleostomy. These surgical steps may be influenced by the anatomical variations that may result in different levels of difficulty during surgery. Accordingly, surgical procedure should be modifiable, if necessary, to obtain adequate surgical exposure.

received

August 2, 2017

accepted

March 9, 2018

published online

May 9, 2018
High-resolution computed tomography (HRCT) has always been the gold standard for the preoperative assessment of the cochlear implant $(\mathrm{CI})$ patients and can predict the difficulty of the surgical procedure., ${ }^{1,2}$

The aim of our study is to provide a basic structured format for the preoperative reading of the CT scan in relation to each of the key surgical steps, which can be tailored to meet the individual requirements. The proposed reporting format, in the form of a simple checklist, follows the order in

Copyright (e 2019 by Thieme Revinter Publicações Ltda, Rio de Janeiro, Brazil 
which the ear structures are approached during surgery and allows for the proper preoperative assessment.

\section{Patients and Methods}

\section{Ethical Considerations}

Both the research project and patients' records retrieval were approved by the institutional review board (IRB). The patients' consents for using their data in the research project were also obtained.

\section{Study Design}

This was a prospective cohort study of consecutive cochlear implant surgeries performed at our tertiary implant center in the period from January 2014 to December 2016. Patients with inner ear anomalies, previous mastoid surgery, reimplantations, revision surgeries and patients who were implanted via alternative techniques (for example, transcanal approach) were excluded from the study.

\section{Subjects}

Forty-seven cases have fulfilled the inclusion criteria and were included in the study. All patients were having bilateral severe-to-profound or profound sensorineural hearing loss.

\section{Methods}

High Resolution Computed Tomography (HRCT) scans of the temporal bone were obtained with slice thickness of $1.0 \mathrm{~mm}$ and were acquired at $120 \mathrm{kVp}, 250 \mathrm{~mA}$, imaging matrix of $512 \times 512,160 \mathrm{~mm}$ field of view (FOV) and voxel size of $\approx$ $0.31 \frac{1}{2} \times 0.31 \frac{1}{2} \times 0.35 \mathrm{~mm}^{3}$. They were viewed in the standard bone window setting and were analyzed and reported according to our proposed radiology checklist (-Fig. 1).

All images were evaluated by the primary author. To avoid interobserver variability, the images were re-evaluated retrospectively by another independent investigator. When the evaluation by the two investigators was different, the final decision was made by the two investigators reviewing the scan together. Each item of the proposed checklist was assessed objectively as follows:

1. Mastoid evaluation: feasibility of cortical mastoidectomy depends on the location of the sigmoid sinus (SS) and the level of the temporal dura. The SS locations were classified into four types according to their relation to three imaginary lines; line 1) the posteriorly-extended line that joins the common crus of the posterior semi-circular canal (PSCC) to the PSCC; line 2) the posteriorly-extended line of the tympanic segment of the facial nerve (FN); and line 3 ) the posteriorly-extended line of the malleal-incudal axis (-Fig. 2). ${ }^{3}$ Types 3 and 4 were considered as predictors for contracted mastoid and difficult mastoidectomy. The dural level was measured relative to the upper border of the petrous bone (-Fig. 3). ${ }^{4}$

2. Posterior tympanotomy \& cochleostomy evaluation: the presence of air cells around the facial recess (sentinel air cell) was assessed using the axial cuts. The impact of the FN course on the feasibility of posterior tympanotomy as well as the accessibility of the round window (RW), with subsequent feasibility of cochlear implant (CI) electrode array insertion, was assessed using the proposed prediction line by Mandour et $\mathrm{al}^{5}$ (-Fig. 4 ).

The difficulties encountered with each of the three key intraoperative steps (cortical mastoidectomy, posterior

\section{CI pre-operative Radiologic Rapid Checklist}
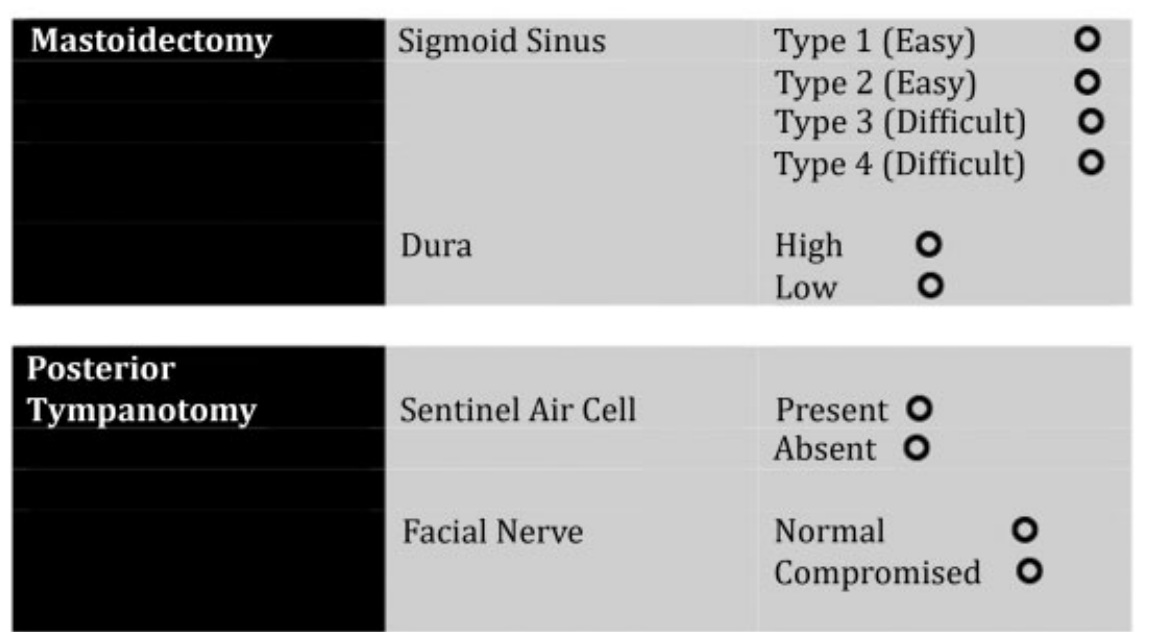

$\begin{array}{ll}\text { Sentinel Air Cell } & \begin{array}{l}\text { Present } \\ \text { Absent } \mathbf{0}\end{array} \\ & \\ \text { Facial Nerve } & \begin{array}{l}\text { Normal } \\ \text { Compromised }\end{array}\end{array}$

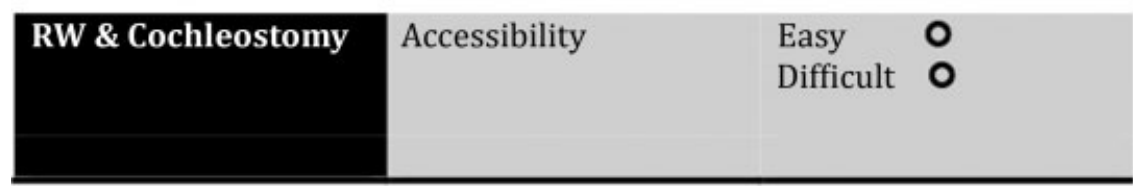

Fig. 1 Radiological checklist used for reporting $\mathrm{Cl}$ cases. 


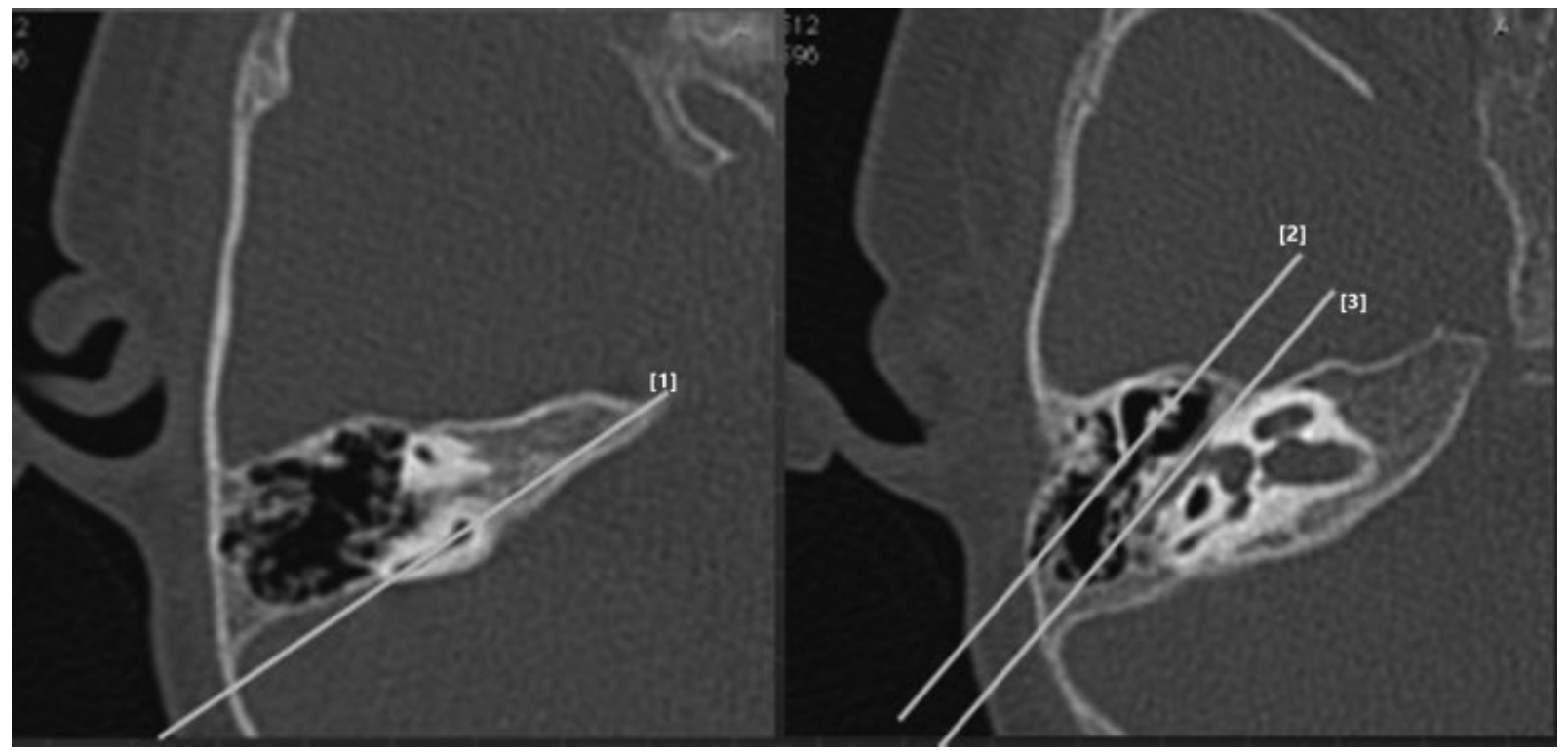

Fig. 2 Two axial HRCT sections. Based on the shown reference lines, the sigmoid sinus was categorized into four types; type [1] the most protruding portion of the sigmoid sinus is medial or posterior to line 1, type [2] the most protruding portion of the sigmoid sinus is located between line 1 and line 2, type [3] the most protruding portion of the sigmoid sinus is located between line 2 and line 3 , and type 4 ) the most protruding portion of the sigmoid sinus is lateral or anterior to line 3.

tympanotomy, and round window access) were scored subjectively according to the following scale: 1 (easy) - indicated a classic straightforward surgical step; 2 (moderate) - indicated some difficulty that could be simply overcome by positional manipulation of the patient or the microscope; and 3 (difficult) - indicated definite difficulty interfering with optimum achieving of the surgical step in spite of positional manipulation or that required special instrumentation to accomplish the step. Scoring was performed by the primary surgeons who were blinded to the potential predictors of the difficulties reported on the checklist.

\section{Statistical Analysis}

Using a two-sided $Z$ test and $\alpha$ level of 0.05 , a power analysis showed that recruiting a sample size of 47 patients will yield an $80 \%$ power to detect the difference in the predictability of the surgical difficulties with and without utilizing our checklist.

The chi-square test of independence was used to find the potential relationship between the difficulty of the mastoidectomy and the SS location as well as the potential relationship between the difficulty in accessing the facial recess and the presence/absence of the facial recess air cell. It was also used to determine the potential relationship between the difficulties in RW accessibility encountered during surgery, which were reported radiologically.

One-way analysis of variance (ANOVA) and Student $t$-test were used to compare the level of the tegmen between the three groups representing the degrees of difficulties of the mastoidectomy (that is, easy, moderate, and difficult).

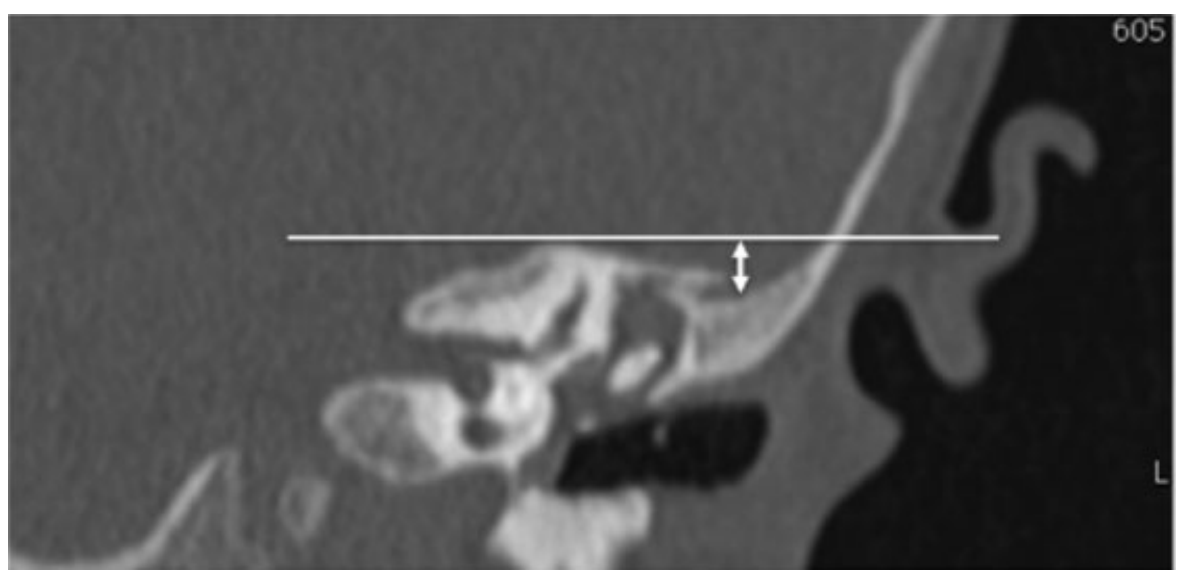

Fig. 3 Coronal cut of HRCT illustrating the distance measured from a line drawn tangentially from the upper edge of the petrous bone to the lowest point of temporal dura. 

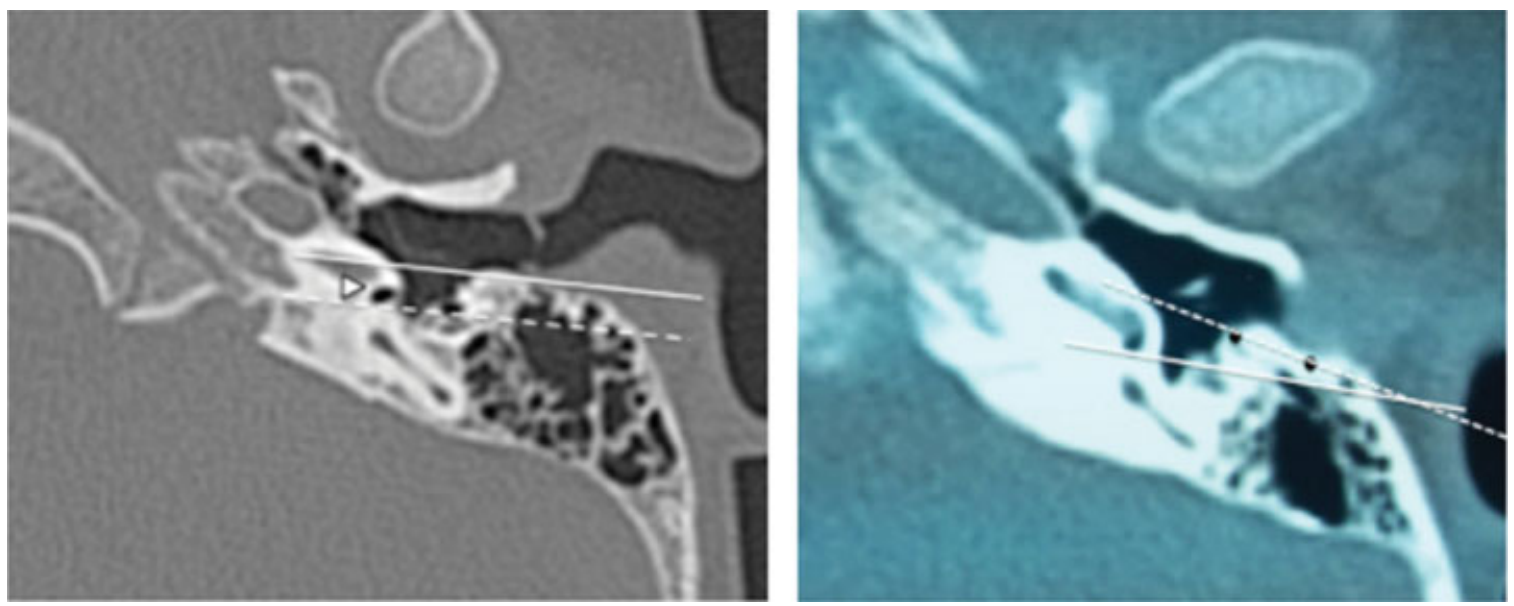

Fig. 4 Two axial HRCT sections. The left section shows round window area (triangle). The EAC line (solid line) was drawn between the bonycartilaginous junction of the posterior EAC wall and the tympanic annulus, and the RW line (dashed line) was drawn from the posterior edge of the RWN along the anterolateral part of the FN. Notice that both lines are nearly parallel, indicating good RW accessibility. The right image shows that the EAC line (dashed line) and the RW line (solid line) intersect with each other laterally, indicating limited accessibility.

\section{Results}

Forty-seven patients were included in the study (29 male and 18 female). They were 41 children with a mean age of 3.7 years (range: from 9 months to 8.5 years) and 6 adults with a mean age of 22.4 years (range: from 19 to 40 years). All the children were prelingual while five adults were postlingual, and one was perilingual. A nucleus freedom cochlear implant system with CI422 electrode (Cochlear, Lane Cover, NSW, Australia) was used in 32 patients, and an Advanced Bionics HiRes 90K system with Hifocus 1J electrode (Sylmar, California, USA) was used in the remaining 15 patients. Fortyfive cases had hearing loss of unknown etiology, while in one adult hearing loss was posttraumatic and, in another case, it was due to autoimmune inner ear disease. Forty patients received a $\mathrm{Cl}$ on the right side, and seven patients on the left side. There was no statistically significant correlation between neither age nor sex and radiological measurements or surgical findings.

Regarding the dural level, the mean distance of tegmen from the tangential line through the upper edge of the petrous bone was different between the three groups (difficult $=7.5 \pm 0.22 \mathrm{~mm}$, moderate $=5.36 \pm 0.46 \mathrm{~mm}$, and easy $=4.48 \pm 0.35 \mathrm{~mm}$ ). The mean distance of the difficult group was significantly longer than that of the easy group based on one-way ANOVA ( - Table 1 ).

Table 1 Correlation between radiologically predicted level of temporal dura and surgical difficulty scales encountered during mastoidectomy.

\begin{tabular}{|l|l|l|l|}
\hline \multirow{2}{*}{ Dural level } & \multicolumn{3}{|l|}{ Surgical difficulties } \\
\cline { 2 - 4 } & Easy & Moderate & Difficult \\
\hline High & 17 & 10 & 0 \\
\hline Low & 0 & 0 & 20 \\
\hline
\end{tabular}

A chi-square test of independence showed that there was a significant association between the difficulty encountered during the cortical mastoidectomy and the SS type, as reported in the radiology checklist $(p$-value $<0.001)$ (-Table 2). The absence of an air cell around the FN was associated significantly with difficulty in accessing the facial recess ( $p$-value $<0.001$ ). Also, it showed a significant association between the difficulties in RW accessibility encountered during surgery, which were reported radiologically using the proposed prediction line $(p$-value $<0.001)$ (-Fig. 5).

\section{Discussion}

There is a high degree of variability between surgeons in analyzing the preoperative CT scan. Our goal is to formalize and standardize the various radiological parameters to provide a basic structured format for reporting the preoperative $\mathrm{CT}$ in a checklist form. The reported checklist allows proper systematic surgical evaluation and follows the order in which the ear structures are approached during surgery.

In our study, we reported that contracted mastoid cavity and low-lying tegmen were associated with a greater degree

Table 2 Correlation between type of mastoid pneumatization, determined radiologically, and surgical difficulty scales encountered during mastoidectomy

\begin{tabular}{|l|l|l|l|}
\hline \multirow{2}{*}{$\begin{array}{l}\text { Mastoid pneumatization } \\
\text { type } \\
\text { (n. of cases) }\end{array}$} & \multicolumn{3}{|l|}{ Surgical difficulties } \\
\cline { 2 - 4 } & Easy & Moderate & Difficult \\
\hline Type 1 (11 cases) & 10 & 1 & 0 \\
\hline Type 2 (8 cases) & 6 & 1 & 1 \\
\hline Type 3 (10 cases) & 1 & 3 & 6 \\
\hline Type 4 (18 cases) & 0 & 5 & 13 \\
\hline
\end{tabular}




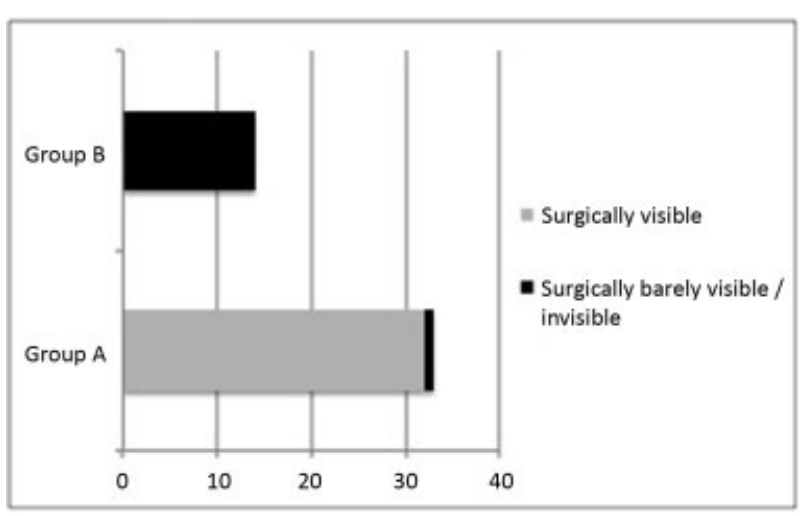

Fig. 5 Correlation between predicted and intra-operative RWN visibility (group A represents radiologically visible RWN, group B represents radiologically invisible RWN)

of difficulty during mastoidectomy with difficult identification of the important landmarks required for subsequent steps (such as the lateral semicircular canal and incus), which may result in high risks of iatrogenic injury to the FN and labyrinth. 6

We have noticed that the anteriorly displaced SS may interfere with proper visualization on turning the head of the patient away from the surgeon's side, which may be needed to expand the surgical field view to identify the various landmarks, such as the short process of incus and the tympanic segment of FN.

Our results seem to be consistent with previous studies in the literature. Pereira et $\mathrm{al}^{4}$ have reported that surgical difficulties during mastoidectomy were encountered when the distance between the anterior border of the SS and the posterior wall of the EAC was less than $9 \mathrm{~mm}$, and the distance between the temporal meninges and the upper edge of the petrous bone was equal to or above $7 \mathrm{~mm}$. Park et $\mathrm{al}^{6}$ reported that low-lying tegmen and poor mastoid pneumatization were associated with a greater difficulty during mastoidectomy.

Drilling in the area of sentinel air cells (air cells around the facial recess) exposes the top end of the facial recess. ${ }^{7}$ In our study, the absence of sentinel air cells on the preoperative CT scan was associated with a higher degree of difficulty during posterior tympanotomy. This finding was similar to that observed by Park et al $^{6}$

It has been widely reported in the literature that the angle between the cortex of the EAC and the FN, and FN location are the most important variables with strongest correlation to round window niche (RWN) accessibility with subsequent feasibility of electrode array insertion. ${ }^{8,9}$ Apart from complex radiologic measurements, we have utilized the prediction line method proposed by Mandour et $\mathrm{al}^{5}$ to assess RWN accessibility. We have found a significant correlation between predicted radiologic difficulties and actual surgical difficulties encountered during operation.
Other factors that could compromise the feasibility of electrode array full insertion, as anomalous cochlea and abnormal cochlear patency, have been considered but have not been reported in our proposed checklist as those cases did not fulfill the inclusion criteria of our study. These cases were managed individually and were excluded from the study to avoid bias.

We recognize that there are some limitations in our study, including that the other factors that may determine round window visibility have not been tested and assessment of the difficulty of each surgical step is subjective. These issues shall be addressed in future studies.

\section{Conclusion}

The proposed checklist provides a systematic structured approach for preoperative radiological evaluation. Contracted mastoid and lower tegmen position were associated with a greater difficulty of the cortical mastoidectomy. The presence of an air cell around the facial nerve was predictive of easier facial recess access exposure. Facial nerve location and EAC posterior wall inclination were predictive of difficult RW accessibility.

\section{Conflicts of Interest}

The authors declare that there are no conflicts of interest.

\section{References}

1 Vaid S, Vaid N. Imaging for cochlear implantation: structuring a clinically relevant report. Clin Radiol 2014;69(07):e9-e24

2 Bettman RH, Van Olphen AF, Zonneveld FW, Huizing EH. Preoperative imaging protocol for cochlear implant candidates. Acta Otolaryngol 2004;124(09):1028-1032

3 Sun DI, Lee DH, Jang KH, et al. A suggested new classification system for the anatomic variations of the sigmoid sinus: a preliminary study. Int Adv Otol 2009;5:1-5

4 Pereira AR Júnior, Pinheiro SD, Vieira de Castro JD, et al. Mastoidectomy: anatomical parameters x surgical difficulty. Int Arch Otorhinolaryngol 2012;16(01):57-61

5 Mandour MF, Khalifa MA, Khalifa HA, Tomoum MO. A novel radiologic check test of round window accessibility for cochlear implantation: Our experience in 198 cases. Clin Otolaryngol 2017;42(05):1108-1111

6 Park E, Amoodi H, Kuthubutheen J, Chen JM, Nedzelski JM, Lin VY. Predictors of round window accessibility for adult cochlear implantation based on pre-operative CT scan: a prospective observational study. J Otolaryngol Head Neck Surg 2015;44:20

7 Cooper HR. Selection criteria and prediction of outcomes. In: Huw R, Cooper LCC, editor. Cochlear Implants: A Practical Guide. Second Edition ed. London and Philadelphia: Whurr Publishers; 2006:132-150

8 Lee DH, Kim JK, Seo JH, Lee BJ. Anatomic limitations of posterior tympanotomy: what is the major radiologic determinant for the view field through posterior tympanotomy? J Craniofac Surg 2012;23(03):817-820

9 Kashio A, Sakamoto T, Karino S, Kakigi A, Iwasaki S, Yamasoba T. Predicting round window niche visibility via the facial recess using high-resolution computed tomography. Otol Neurotol 2015;36(01):e18-e23 